\title{
The Natural History of Pneumonic Tularemia in Female Fischer 344 Rats after Inhalational Exposure to Aerosolized Francisella tularensis Subspecies tularensis Strain SCHU S4
}

\author{
Julie A. Hutt, ${ }^{*}$ Julie A. Lovchik, ${ }^{\dagger \dagger}$ Alexander Dekonenko, ${ }^{\dagger}$ Andrew C. Hahn, ${ }^{\dagger}$ and Terry H. $\mathrm{Wu}^{\dagger \dagger}$
}

From the Lovelace Respiratory Research Institute, ${ }^{*}$ Albuquerque; the Center for Infectious Disease and Immunity ${ }^{\dagger}$ and the Department of Internal Medicine, ${ }^{\ddagger}$ The University of New Mexico Health Science Center, Albuquerque, New Mexico

Accepted for publication September 22, 2016.

Address correspondence to Julie A. Hutt, D.V.M., Ph.D., Lovelace Respiratory Research Institute, 2425 Ridgecrest Dr SE, Albuquerque, NM 87108. E-mail: jhutt@1rri.org.

\begin{abstract}
The inbred Fischer 344 rat is being evaluated for testing novel vaccines and therapeutics against pneumonic tularemia. Although primary pneumonic tularemia in humans typically occurs by inhalation of aerosolized bacteria, the rat model has relied on intratracheal inoculation of organisms because of safety and equipment issues. We now report the natural history of pneumonic tularemia in female Fischer 344 rats after nose-only inhalational exposure to lethal doses of aerosolized Francisella tularensis subspecies tularensis, strain SCHU S4. Our results are consistent with initial uptake of aerosolized SCHU S4 from the nasal cavity, lungs, and possibly the gastrointestinal tract. Bacteremia with hematogenous dissemination was first detected 2 days after exposure. Shortly thereafter, the infected rats exhibited fever, tachypnea, and hypertension that persisted for 24 to 36 hours and then rapidly decreased as animals succumbed to infection between days 5 and 8 after exposure. Tachycardia was observed briefly, but only after the core body temperature and blood pressure began to decrease as the animals were near death. Initial neutrophilic and histiocytic inflammation in affected tissues became progressively more fibrinous and necrotizing over time. At death, as many as $10^{10}$ colony-forming units were found in the lungs, spleen, and liver. Death was attributed to sepsis and disseminated intravascular coagulation. Overall, the pathogenesis of pneumonic tularemia in the female F344 rat model appears to replicate the disease in humans. (Am J Pathol 2017, 187: 252-267; http://dx.doi.org/10.1016/j.ajpath.2016.09.021)
\end{abstract}

Tularemia is an uncommon zoonotic disease caused by the pleomorphic, Gram-negative coccobacillus, Francisella tularensis subspecies tularensis. Naturally occurring tularemia in humans manifests in multiple forms, depending on the route of exposure to the bacteria. The ulceroglandular form develops after skin infection following direct contact with an infected animal or bite of an infected insect vector; the occuloglandular form develops after bacterial contamination of the eye; the oropharyngeal or gastrointestinal form develops after ingestion of contaminated food or water; and the inhalational form develops after inhalation of contaminated respirable particles. The disease typically manifests in humans as inflammation and ulceration of the skin or mucosal surface at the portal of entry, followed by marked suppurative to pyogranulomatous and necrotizing inflammation in the draining lymph nodes. ${ }^{1}$ Left untreated, hematogenous spread after any route of exposure results in inflammation within the liver, spleen, bone marrow, and lungs. Occasionally, a portal of entry is not identified, and the disease manifests as an enlarged, inflamed lymph node (glandular form) or as septicemia (typhoidal form).

$F$. tularensis is one of the most highly infectious, pathogenic bacteria known to humans. Under controlled conditions, inhalation of 10 to 52 organisms of the prototype

\footnotetext{
Supported by the National Institute of Allergy and Infectious Diseases, NIH, Department of Health and Human Services contract HHSN 272201000037I-HHSN27200001, Task Order A25 (T.H.W.), and the Defense Threat Reduction Agency contract HDTRA1-12-C-0046 (T.H.W.).

Disclosures: None declared.
} 
subspecies tularensis strain SCHU S4 caused debilitating disease in humans that required prompt antibiotic treatment. ${ }^{2}$ Illness appeared between 4 and 7 days after exposure and was characterized by flu-like symptoms, including fever, headache, nausea, myalgia, malaise, and nonproductive cough. Higher-exposure doses of up to 25,000 organisms of SCHU S4 were routinely used for vaccine and therapeutic studies, and the resulting disease tended to have a shorter incubation period but similar disease presentation as the lower doses. ${ }^{3,4}$ Because human subjects in these clinical studies were always treated with antibiotics soon after the onset of clinical disease, details about the progression and late-stage disease relied mostly on reports of natural infection with no information on exposure dose. Before the advent of effective antibiotic therapy, $30 \%$ to $60 \%$ of patients who developed naturally occurring pleuropulmonary tularemia died of this disease. ${ }^{5}$

Because of the high degree of infectivity and virulence, and the ease of bacterial dissemination by the aerosol route, F. tularensis was developed as a bioweapon during World War II. ${ }^{6-8}$ During the 1950 s and 1960s, the US military developed techniques to disseminate $F$. tularensis as an aerosol, but terminated its biological weapons development program in the early 1970 s. $^{7}$ Current military and nonmilitary research on tularemia in the United States is focused on defensive medical research to develop improved diagnostic, prophylactic, and therapeutic strategies for the medical management of civilian and military personnel after the use of $F$. tularensis as a biological weapon. ${ }^{9}$

Currently, there is no licensed tularemia vaccine, and frontline antibiotics may become ineffective if the bacteria develop natural or engineered resistance. Therefore, development of novel vaccines and therapeutics is a high priority in the national biodefense program. Animal models that accurately reproduce the human disease are essential in the process of developing new vaccines and therapeutics against tularemia. Because of the low natural incidence of tularemia, proven efficacy in a well-characterized animal model will also be necessary to satisfy the Food and Drug Administration's Animal Efficacy Rule (21 CFR 314.610, 21 CFR 601.9) for licensure of any new tularemia countermeasure.

Several studies have examined the pathogenesis of primary pneumonic tularemia after inhalation of $F$. tularensis aerosols in nonhuman primates and identified nonhuman primate species in which the disease pathogenesis closely resembles that of humans. ${ }^{10-16}$ However, monkeys are expensive and difficult to work with, and many facilities are not equipped to house and perform aerosol exposures on large animal species. Thus, a small animal model that recapitulates the human disease and is predictive of the human response to vaccination is needed to screen candidate vaccines and therapeutics.

Multiple small animal species have been used to test the efficacy of vaccines and therapeutics against tularemia. ${ }^{17}$ Many studies have been performed in mice. However, mice are highly susceptible to $F$. tularensis, including strains that are of low virulence in humans, such as the live vaccine strain (LVS) ${ }^{18}$ New Zealand white rabbits have been characterized as an animal model that closely recapitulates primary pneumonic tularemia in humans. However, in contrast to humans, vaccination of rabbits with LVS does not protect from death after aerosol exposure to F. tularensis. ${ }^{2,19-22}$

Previously published data demonstrate that Fischer 344 (F344) rats show a similar susceptibility to $F$. tularensis strains as has been reported for humans. ${ }^{23,24}$ F344 rats were sensitive to an intratracheal inoculation of the highly virulent SCHU S4 strain of $F$. tularensis [lethal dose $50 \%$ $\left(\mathrm{LD}_{50}\right)<200$ ], but were resistant to infection with subspecies holarctica (strain OR96-0246, $\mathrm{LD}_{50}=10^{5}$ ) and subspecies novicida ( (train $\mathrm{U} 112, \mathrm{LD}_{50}=5 \times 10^{6}$ ). Preliminary studies also suggested that SCHU S4 infection causes similar physiological responses in F344 rats and humans, including elevated temperature, respiratory rate, and blood pressure. In addition, F344 rats have been shown to have similar responses to immunization with LVS as humans. . $23,25^{2}$

The objective of the current natural history studies described in this report was to characterize the pathogenesis of primary pneumonic tularemia in female F344 rats exposed to aerosolized $F$. tularensis, SCHU S4. A secondary goal was to identify an early, reliable indicator of infection that could be used as a treatment trigger point in future efficacy studies to evaluate novel potential therapeutics against $F$. tularensis.

\section{Materials and Methods}

\section{Animals}

Specific pathogen-free female F344 rats, weighing between 150 and $180 \mathrm{~g}$ and at approximately 4 months of age, were purchased from Harlan Laboratories (Indianapolis, IN). The animals were housed individually in ventilated cages. The cages were lined with Tek-Fresh pelleted, virgin paper fiber bedding and maintained at negative pressure to contain F. tularensis. The animals were fed ad libitum with Teklad irradiated rodent chow 2918 and given autoclaved, chlorine dioxide-treated water.

\section{Surgical Implantation of Telemetry Transmitters}

Two models of telemetry transmitters (DSI, New Brighton, $\mathrm{MN}$ ) were used: model C50-PT measured blood pressure, core body temperature, respiratory rate, heart rate, and activity level; and model TA10TA-F40 measured core body temperature and activity level. Aseptic surgical techniques were used to implant telemetry transmitters within the peritoneal cavity. Rats were anesthetized with $4 \%$ to $5 \%$ isoflurane/oxygen at a flow rate $2 \mathrm{~L} /$ minute and maintained with $1.5 \%$ to $2 \%$ isoflurane/oxygen during surgery. The animals were kept warm over a heating pad during surgery. The abdomen was shaved between the xiphoid process and 
a point $2 \mathrm{~cm}$ cranial to the genital opening, and the skin was wiped three times with betadine (Purdue Products, Stamford, CT). A midline skin incision was made with a surgical scalpel from $1 \mathrm{~cm}$ below the xiphoid process to $3 \mathrm{~cm}$ cranial to the genital opening, followed by an incision through the abdominal wall using small scissors. To insert the pressure catheter on model C50-PT, the abdominal aorta was ligated at the level of the left renal artery and at the iliac bifurcation using surgical suture under light tension. A small incision was made in the aorta $1 \mathrm{~cm}$ rostral to the iliac bifurcation using the tip of a 20-gauge hypodermic needle. The pressure catheter was then threaded through the incision until it reached the rostral occlusion site of the aorta. The catheter was lightly glued into place at the site of the aortic incision using Vetbond (3M, St. Paul, MN) adhesive. The ligations were removed, and the aorta was checked along its length to ensure the absence of bleeding. The transmitter body was placed adjacent to the abdominal wall and ventral to the small intestines. The abdominal wall incision was closed using Vicryl (Ethicon, Cincinnati, OH) sutures with the central sutures passing through suture loops located on the transmitter body to secure the transmitter. The skin was closed using a combination of staples and cyanoacrylate adhesive (Loctite, Westlake, $\mathrm{OH}$ ). Anesthesia was discontinued, and a single dose each of penicillin $\mathrm{G}(1 \mathrm{mg} / 100$ $\mathrm{g}$ body weight) and buprenorphine $(0.5 \mathrm{mg} / 100 \mathrm{~g}$ body weight) were administered intramuscularly. Staples were removed approximately 7 days after surgery.

\section{Surgical Implantation of Jugular Vein Catheters}

Rats were treated before surgery with $60,000 \mathrm{U} / \mathrm{kg}$ penicillin $\mathrm{G}$ benzathine and $60,000 \mathrm{U} / \mathrm{kg}$ penicillin $\mathrm{G}$ procaine (Combi-Pen-48; Bimeda Inc., Le Sueur, MN), 0.06 mg/kg buprenorphine, and $10 \mathrm{~mL}$ saline by s.c. injection. Rats were anesthetized with $4 \%$ to $5 \%$ isoflurane/oxygen at a flow rate $2 \mathrm{~L} /$ minute and maintained at surgical anesthesia plane with $1.5 \%$ to $2 \%$ isoflurane/oxygen. A sterile polyurethane catheter (SAI Infusion Technologies, Lake Villa, IL) was inserted into the jugular vein just before the right atrium. The catheter was anchored and exteriorized through a skin incision in the dorsal nape of the neck and connected to a vascular access harness (Instech, Plymouth Meeting, PA). The catheter and vascular access port were flushed with $200 \mu \mathrm{L}$ of sterile saline for injection and locked with $100 \mu \mathrm{L}$ of heparin/glycerol catheter lock solution (SAI Infusion Technologies) immediately after surgery. The rats were wrapped with self-adherent wrap (3M, St Paul, MN) to protect the catheter and harness during the study. A second dose of buprenorphine was administered the next day, and the catheters were flushed again.

\section{Telemetry Data Acquisition}

Telemetry signals were acquired for 30 seconds every 10 to 15 minutes and analyzed using Dataquest ART (DSI).
Blood pressure and core body temperature were measured directly. Heart rate and respiration rate were calculated from the pressure form.

\section{Preparation of Organism}

The F. tularensis subspecies tularensis, SCHU S4 strain used for these studies was originally derived from Master Cell Bank, NR-28534 (BEI Resources, Manassas, VA). The Master Cell Bank had been subcultured in modified cystine partial hydrolysate broth once to produce a submaster stock and a second time to produce a working stock; aliquots of each were stored in $20 \%$ glycerol at $-80^{\circ} \mathrm{C}$. Before each aerosol exposure, a vial from the SCHU S4 working stock was thawed to room temperature, plated onto cystine heart agar with rabbit blood, $100 \mathrm{U} / \mathrm{mL}$ penicillin $\mathrm{G}$, and $100 \mathrm{U} / \mathrm{mL}$ polymixin B (CHAB, Thermo Fisher Scientific Remel Products, Lenexa, KS), and incubated for 3 to 5 days at $37^{\circ} \mathrm{C}$. The day before exposure, approximately 10 individual colonies of 1 to $2 \mathrm{~mm}$ in diameter were suspended into $4.5 \mathrm{~mL}$ Chamberlain's chemically defined medium (Teknova, Hollister, CA) to produce a bacterial suspension with an OD at $600 \mathrm{~nm}$ $\left(\mathrm{OD}_{600}\right)$ of $0.1 \pm 0.01$ [equivalent to approximately $10^{8}$ colony-forming units (CFUs) per milliliter]. A $400-\mu \mathrm{L}$ aliquot of the $\mathrm{OD}_{600}$ normalized suspension was inoculated into a sterile $500 \mathrm{~mL}$ flask containing $100 \mathrm{~mL}$ sterile Chamberlain's chemically defined medium. A second flask containing only $100 \mathrm{~mL}$ of sterile Chamberlain's chemically defined medium was used to confirm sterile conditions during culture. The flasks were incubated at $37^{\circ} \mathrm{C} \pm 2^{\circ} \mathrm{C}$ with $200 \mathrm{rpm}$ rotary aeration for 22 to 25 hours to achieve an $\mathrm{OD}_{600}$ range of 0.2 to 0.8 . An aliquot of the overnight culture was then serially diluted in sterile brain heart infusion broth (Teknova) to 1 to $5 \times 10^{6} \mathrm{CFU} / \mathrm{mL}$. The nebulizer suspension was stored on ice until it was loaded into the generator cup and used to produce aerosols. Preliminary data showed that SCHU S4 viability did not change after incubating on ice for 5 hours.

\section{Aerosol Exposure}

Aerosolized SCHU S4 was generated using a Collison 3-jet nebulizer (BGI, Inc., Waltham, MA) and presented to the study animals in a nose-only exposure chamber (In-Tox Products, Inc., Moriarty, NM). The generator flow rate was maintained at $7.5 \mathrm{~L} /$ minute, and the impinger flow rate was maintained at $5 \mathrm{~L} /$ minute. The animals were exposed to aerosolized SCHU S4 for 15 minutes, and the system was purged for 2 minutes before the animals were removed. Particle sizes were measured for 10 minutes into each exposure run by drawing an aerosol sample for 20 seconds. The aerosol samples were diluted 1:20 using an aerosol dilutor (TSI, Shoreview, MN) and analyzed using an aerodynamic particle sizer spectrometer (TSI). Most aerosol particles were within the 1- to 5- $\mu \mathrm{m}$ range required for deep lung deposition. There were also nonrespirable particles $>5$ $\mu \mathrm{m}$ that were caused by coagulation of aerosol droplets; 
these represented $<15 \%$ of the aerosolized particles by mass. A sample from the all glass impinger (Ace Glass, Inc., Vineland, NJ) was plated after each run onto selective cystine heart agar plates (Thermo Fisher Scientific Remel Products, Lenexa, KS), and the impinger concentration was used to calculate the aerosol concentration. The lung deposition was determined by plating lung homogenates from a subset of rats euthanized 1 hour after termination of each exposure run. The purity of aerosolized samples was assessed by examination of colony morphology.

\section{Clinical Observations}

Clinical observations were performed once a day before exposure and twice a day after exposure. Clinical observations were assigned a score between 0 and 4: 0 , bright, alert, responsive, and active; 1 , mild clinical signs of infection, such as coat ruffling and porphyrin secretion; 2, pronounced decrease in activity and responsiveness to stimulation, ruffled coat, and rapid and shallow breathing; 3, moribund (eyes closed, completely labored breathing, inactive, and unresponsive to tail tug; moribund rats were euthanized when their core body temperature was $30^{\circ} \mathrm{C}$ or lower); 4 , dead.

\section{Necropsy}

Euthanasia was performed by i.p. injection of at least 200 $\mathrm{mg} / \mathrm{kg}$ sodium pentobarbital solution. Necropsies were conducted on all animals at scheduled or moribund euthanasia or within 12 hours of death for animals that were found dead. The necropsy included an examination of external surfaces and orifices, extremities, and the abdominal, thoracic, and cranial cavities and their contents. Before initiation of the necropsy, blood was obtained from animals undergoing euthanasia by open cardiocentesis while under a surgical plane of anesthesia. Blood and a portion of the lungs, liver, and spleen were collected aseptically for quantitative culture at the time of necropsy. Portions of the lungs, liver, spleen, nasal cavity, oropharynx, kidneys, adrenal glands, bone marrow (sternum), heart, brain, and lymph nodes draining the thoracic cavity and head were routinely collected and fixed in 10\% neutral-buffered formalin. Other organs were also collected and fixed in $10 \%$ neutral-buffered formalin if macroscopic findings were identified at necropsy or based on clinical signs. Necropsy findings were documented using a brief morphological description of all macroscopic abnormalities. Whole organ weights (lungs, liver, spleen, kidneys, heart, and brain) and cultured portion organ weights (lungs, liver, and spleen) were recorded.

\section{Histopathology}

Tissues to include the nasal cavity, oropharynx, lungs, liver, spleen, kidneys, adrenals, bone marrow (sternum), heart, brain, and lymph nodes draining the thoracic cavity and head, and other tissues with macroscopic lesions were trimmed, histoprocessed to slides by routine methods, and stained with hematoxylin and eosin. Liver sections from a subset of rats were also stained with phosphotungstic acid hematoxylin. The nasal cavities and sternums were decalcified in formic acid solution before trimming. Slides were examined by a board-certified veterinary pathologist (J.A.H.). Gradable histomorphologic findings were assigned a severity score from 0 to 4 ( 0 , no finding; 1 , minimal; 2 , mild; 3 , moderate; and 4 , severe) and, where appropriate, a distribution assessment of focal, multifocal, locally extensive, or diffuse.

\section{Microbiology}

A portion of the lungs, spleen, and liver were aseptically collected at necropsy, weighed, and homogenized in $5 \mathrm{~mL}$ sterile phosphate-buffered saline. Serial dilutions of the homogenates were prepared and plated onto $\mathrm{CHAB}$ to determine the number of $F$. tularensis in the tissue samples. On the basis of the bacterial load in the samples, calculations were made to determine the bacterial load (CFUs) in the whole organ. Whole blood was collected at necropsy and transferred into potassium EDTA $\left(\mathrm{K}_{2}\right.$ EDTA) blood collection tubes. An aliquot was serially diluted in phosphate-buffered saline and plated onto CHAB to determine the number of $F$. tularensis $/ \mathrm{mL}$ of blood.

\section{Laboratory Tests}

Whole blood was collected by open cardiocentesis at necropsy for all laboratory tests, except for lactate, which was collected repeatedly from the same animals through jugular vein catheters. As a control for the hematology, clinical chemistry, $\alpha$-2-macroglobulin, and coagulation assays, blood was collected in the same manner from groups of five to six age-matched, uninfected female F344 rats that were purchased and housed with the exposed rats assigned to study (designated as naïve rats). The blood was transferred into potassium EDTA tubes for hematology, citrate tubes for coagulation panel, and serum separator tubes for the chemistry panel and lactate assay. The blood in potassium EDTA tubes was analyzed using the Forcyte Hematology System (Oxford Science, Oxford, CT). The blood in citrate tubes was centrifuged at $3000 \times g$ for 10 minutes at room temperature, and the blood in serum separator tubes was allowed to clot at room temperature for 30 to 60 minutes before being centrifuged at $3000 \times g$ for 10 minutes at room temperature. Serum and plasma were filtered through 0.22$\mu \mathrm{m}$ syringe tip filters and stored at $-20^{\circ} \mathrm{C}$ until sterility was confirmed by culturing aliquots on agar plates. Coagulation and chemistry panels were analyzed by IDEXX RADIL (Columbia, MO), $\alpha$-2-macroglobulin was assayed by enzyme-linked immunosorbent assay (ABCAM, Cambridge, MA), and lactate level was measured using a lactate assay kit (Sigma-Aldrich, St. Louis, MO). 


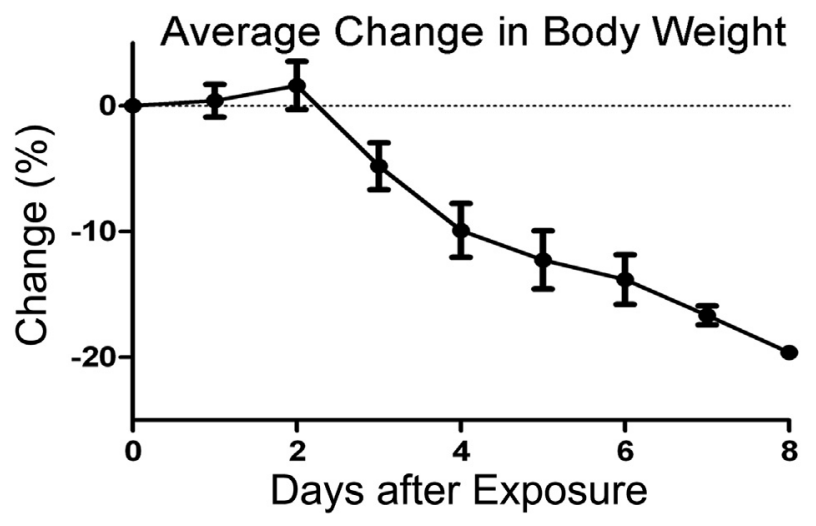

Figure 1 Progressive decreases in body weight of female F344 rats were identified starting on day 3 after exposure to $10^{3}$ CFUs Francisella tularensis, strain SCHU S4. Total body weights were collected on the day of exposure and each day after exposure. The dotted horizontal line represents $0 \%$ change from the baseline weight on the day of exposure. Data are presented as means \pm SD for all rats that were alive on the indicated day.

\section{Results}

$\mathrm{LD}_{50}$ and $\mathrm{LD}_{99}$

Preliminary studies were conducted to determine the $\mathrm{LD}_{50}$ and $\mathrm{LD}_{99}$ of inhaled $F$. tularensis, strain SCHU S4, in female F344 rats. A total of 175 rats in 17 independent studies were exposed to aerosolized SCHU S4, resulting in calculated lung depositions ranging from 0.3 up to 7000
CFUs. Because of the limited sensitivity associated with plating lung homogenates at the low end of the dose range, the $\mathrm{LD}_{50}$ and $\mathrm{LD}_{99}$ and their CIs were first calculated using the generator concentration and then converted to lung deposition using a straight line that estimated the correlation between generator concentration and lung deposition. These rats were monitored for survival for up to 21 days after exposure. From these studies, the $\mathrm{LD}_{50}$ and $\mathrm{LD}_{99}$ were calculated to be 0.7 CFUs (95\% CI, 0.4-1.2 CFUs) and 145.8 CFUs (95\% CI, 35.1-608.9 CFUs), respectively. During the course of these studies, the clinical presentation was similar at all doses, but the time course of infection was shorter for rats exposed to higher doses.

The next studies were designed to fully characterize the disease progression of pneumonic tularemia. Fischer 344 rats were implanted with telemeters and exposed by inhalation of $F$. tularensis aerosols to a lethal, target dose of $10^{3}$ CFUs/rat. A target dose of $10^{3}$ was chosen for these studies to ensure a uniformly lethal dose would be achieved that would allow quantification of actual lung deposition using quantitative microbiology of the lungs from a subset of exposed rats.

\section{Clinical Disease Course and Telemetry}

Multiple studies were performed with rats that had implanted telemeters to allow for monitoring of core body temperature, blood pressure, respiratory rate, and heart rate,
A

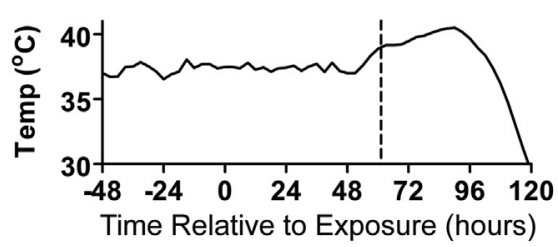

C
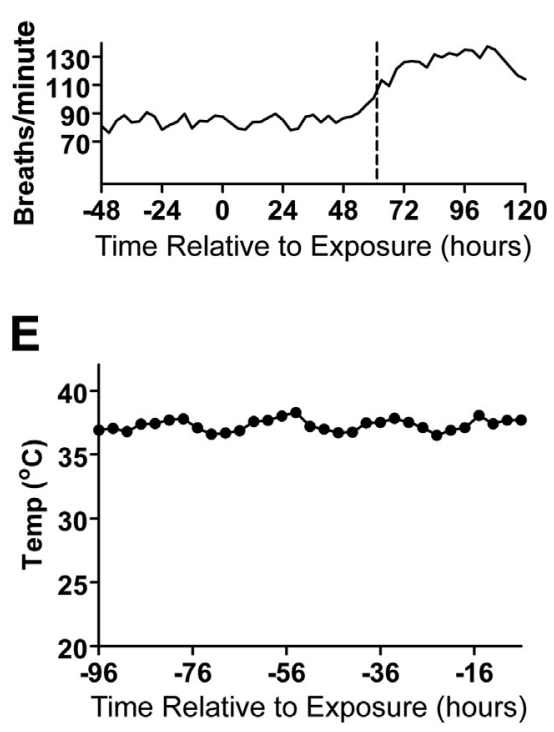

B

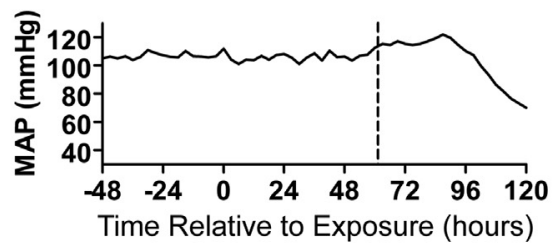

D

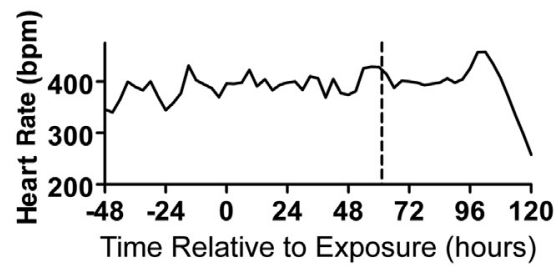

$\mathbf{F}$

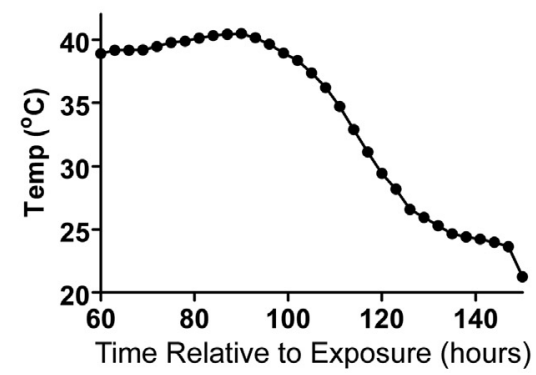

Figure 2 Fever onset was accompanied by increases in mean arterial blood pressure and respiratory rate, but not heart rate. A-D: Telemetry was used to measure changes in core body temperature (temp; $\mathbf{A})$, mean arterial pressure (MAP; B), respiratory rate $(\mathbf{C})$, and heart rate $(\mathbf{D})$. The data from the representative rat shown herein (exposed to 853 CFUs SCHU S4) demonstrate the onset of fever at 61 hours after exposure, as denoted by the dashed vertical lines. Note the lack of increase in heart rate at the onset of fever. $\mathbf{E}$ and $\mathbf{F}$ : Expanded graphs showing core body temperature from the same rat over a 90-hour period before exposure (E) and a 90 -hour period starting at the onset of fever $(\mathbf{F})$. Note the diurnal variation in core body temperature before exposure, and the lack of diurnal variation after the onset of fever. Telemetry data for other rats were similar, with only minor differences in the time of fever onset. bpm, beats per minute. 
Table 1 Francisella tularensis Lung Deposition Dose and Time to Fever Onset

\begin{tabular}{lcll}
\hline Study & $\begin{array}{l}\text { Dose deposited in } \\
\text { lungs, CFUs* }\end{array}$ & $\begin{array}{l}\text { Time to fever, } \\
\text { hours* }\end{array}$ & Rats, $n$ \\
\hline A & $853 \pm 177$ & $64.2 \pm 4.8$ & 6 \\
B & $1234 \pm 290$ & $57.5 \pm 1.1$ & 8 \\
C & $3153 \pm 1095$ & $54.5 \pm 1.8$ & 6 \\
\hline
\end{tabular}

*Data are given as means \pm SD.

CFU, colony-forming unit.

along with clinical signs of infection, and in combination with assessing bacterial dissemination, changes in hematology, clinical chemistry, and histopathology during the entire course of disease. Because rats exposed to the low end of the lethal dose range developed a different distribution of lesions than rats that were exposed to higher doses, the telemetry and pathology results presented herein only include data from rats on studies where the actual exposure dose was $>500$ CFUs/rat.

Overt clinical signs of infection were first detected between days 2 and 3 after exposure, and worsened over time until the rats succumbed to disease or were euthanized in a moribund condition. These were characterized by porphyrin staining of periocular skin, coat ruffling, hunched posture, decreased activity level, and weight loss (Figure 1). In addition, a few rats developed diarrhea as the disease progressed. Rats near death were weak and dyspneic, and exhibited reduced responsiveness to external stimuli.

Fever, defined as a mean core body temperature $>39^{\circ} \mathrm{C}$ for over a 1-hour period, was first detected between days 2 and 3 after exposure and was accompanied by the loss of the normal diurnal pattern of temperature variation (Figure 2, A, $\mathrm{E}$, and F). The average time to fever onset for each of the three studies with telemetry is shown in Table 1. In general, rats that were exposed to higher doses of $F$. tularensis had a shorter incubation period with a more rapid onset of fever. Core body temperatures generally remained elevated for 24 to 36 hours after the onset of fever and then rapidly decreased before animals succumbed to infection between days 5 and 8 after exposure. Changes in mean arterial blood pressures closely paralleled the changes in core body temperature, increasing with the onset of fever, then decreasing as temperatures decreased (Figure 2B). Respiratory rates also increased starting between days 2 and 3 after exposure and only returned to baseline levels when the animals were close to death (Figure 2C). Paradoxically, the heart rate did not increase immediately with the onset of fever, but
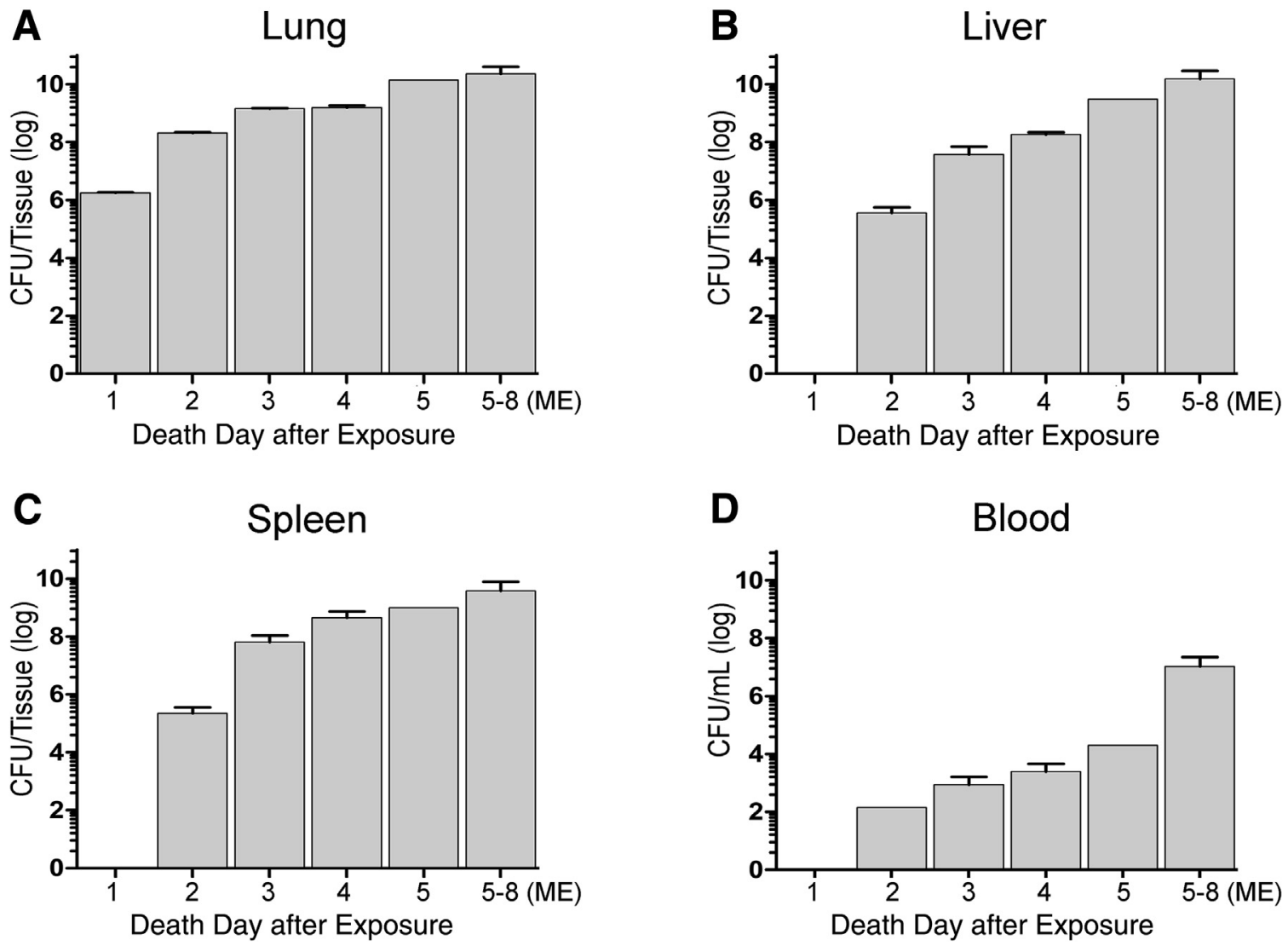

Figure 3 Bacteremia, with bacterial dissemination to the liver and spleen, was first detected on day 2 after exposure to $10^{3}$ CFUs Francisella tularensis, strain SCHU S4. F344 rats were euthanized at predetermined time points between days 1 and 5 after exposure, and when they were identified to be moribund. Portions of the indicated tissues and aliquots of blood were aseptically collected and cultured for $F$. tularensis. Data are presented as the logarithmic transformation of the means \pm SD of bacterial counts (CFUs) from the lungs (A), liver (B), spleen (C), and blood (D). ME, moribund euthanasia. 
decreased slightly from the prefever heart rate, and then increased briefly when the core body temperature and blood pressure started to decrease (Figure 2D). The brief increase in heart rate was followed by a rapid decline in parallel with the declines in core body temperature and mean arterial blood pressure.

\section{Pathology and Microbiology during Disease Progression}

In two of the studies in which telemetry data were captured (1234 and 3153 CFU exposures, respectively), subsets of additional rats were designated for euthanasia on each of the first 5 days (three rats per day) after exposure to characterize the timing of bacterial dissemination to target organs with quantitative microbiology and the changes over time in the macroscopic and microscopic appearance of organs. Blood was also collected from these rats at euthanasia to characterize the clinical pathology changes over time after exposure. Two of the rats assigned to the day 5 euthanasia group died before scheduled euthanasia. The quantitative microbiology, gross, microscopic, and clinical pathology data from the animals euthanized at predetermined time points were combined with the data from the telemetered rats that were monitored during the entire course of disease until death or moribund euthanasia to fully characterize the changes over time.

\section{Microbiology}

Quantitative bacterial cultures from tissues collected on the day after aerosol exposure showed that the infection was still confined to the lungs, but the number of bacteria in the lungs had increased by more than three orders of magnitude compared to the initial exposure deposition of $10^{3} \mathrm{CFUs}$ (Figure 3). Systemic dissemination was observed by day 2 after exposure, preceding the development of fever in the telemetered rats. There were progressive increases over time in the bacterial counts in the lungs, liver, spleen, and blood of infected rats from day 2 after exposure until death. The terminal bacterial burdens ranged between $10^{9}$ and $10^{10}$ CFUs in the lungs and between $10^{8}$ and $10^{10}$ CFUs in the liver and spleen in all animals that succumbed to infection, regardless of the day of death.

\section{Gross Pathology}

Macroscopic findings attributed to aerosol exposure to SCHU S4 were most frequently identified in the lungs, spleen, and lymph nodes draining the head (mandibular) and lungs (tracheobronchial and mediastinal). These are summarized in Supplemental Table S1. Macroscopic findings in the lungs were first noted in rats that were euthanized on day 4 after exposure, and these consisted of multifocal to coalescing, round to irregularly shaped, depressed, and/or dark red areas (Figure 4A) that correlated microscopically with inflammation of the conducting airways and lung parenchyma. Splenic enlargement (Figure 4B) was noted for

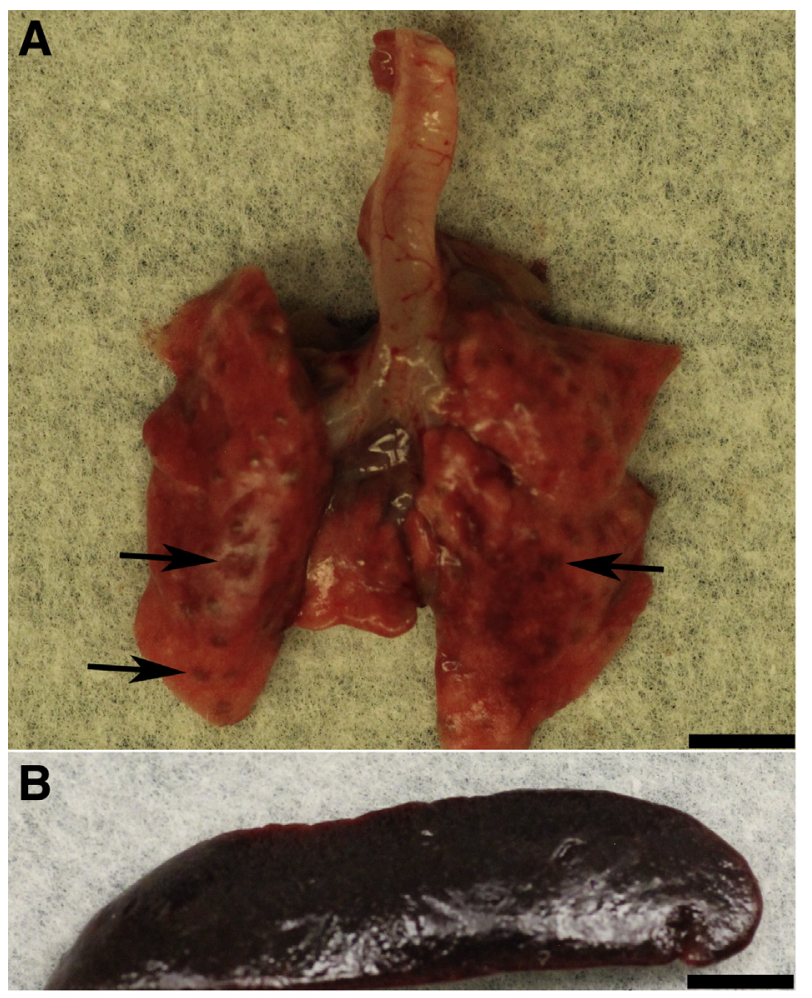

Figure 4 Macroscopic lesions were first detected in the lungs and spleen on day 4 after exposure to $10^{3}$ CFUs Francisella tularensis, strain SCHU S4. A: Lung lesions (arrows) consisted of multifocal to coalescing, round to irregularly shaped, and dark red foci that were depressed from the adjacent lung. B: Spleens were diffusely enlarged. Scale bar $=5 \mathrm{~mm}$ (A and $\mathbf{B})$.

many rats at scheduled euthanasia between days 3 and 5 after exposure and for one rat found dead between days 4 and 5 after exposure. Microscopically, the enlargement generally correlated with histiocytic and neutrophilic inflammation of the splenic red pulp. Interestingly, in the rats that were euthanized because of moribund condition between days 5 and 8 after exposure, and in two-thirds of the rats that were found dead, splenic enlargement was not noted. This was considered to be because of the progressive tissue necrosis in both the red pulp and white pulp in the spleens of SCHU S4-exposed rats over time. The lymph nodes draining the head and lungs (mandibular, tracheobronchial, and mediastinal) of several rats that were euthanized or found dead on day 4 after exposure and later were enlarged, with and without red discoloration, which correlated microscopically with the presence of inflammation.

Additional macroscopic findings were detected sporadically in some rats. The adrenal glands of two rats that were euthanized on day 4 after exposure or later were enlarged and, in one case, discolored red. Microscopically, these observations correlated to histiocytic and fibrinous to necrotizing inflammation with congestion in the adrenal cortex. The thymus of two rats that were euthanized or found dead on day 4 after exposure or later was noted to be reduced in size or undetectable at necropsy. Microscopic 
examination showed reduced numbers of lymphocytes in these thymi, particularly in the cortex. This was considered to be a non-specific, stress-related response in these rats. ${ }^{26}$

\section{Microscopic Pathology}

Microscopic findings by day after exposure are summarized in Supplemental Table S2. Microscopic findings were first detected in the nasal cavity and lungs 2 days after exposure to $F$. tularensis. Lesions in the nasal cavity consisted of histiocytic, neutrophilic, and fibrinous inflammation of the nasal mucosa with exudation of fibrin into the lumen of the nasal cavity. The lung inflammation consisted of histiocytic and neutrophilic infiltrates in bronchioles and adjacent alveoli, bronchi, bronchial-associated lymphoid tissue, and the adventitia of pulmonary vessels. The airway centric inflammation was consistent with aerogenous delivery of bacteria and development of bronchopneumonia.

Three days after exposure, inflammation in the lungs (Figure 5) and nasal cavity increased in severity, and mucous metaplasia of the conducting airway epithelium was evident. In addition, histiocytic to fibrinous/necrotizing inflammation was first identified in the mandibular, tracheobronchial, and mediastinal lymph nodes. Neutrophilic and histiocytic inflammation in the liver and the splenic red pulp were also identified, consistent with lymphohematogenous dissemination of SCHU S4 to these organs. Increased myeloid cells were identified in the bone marrow, consistent with increased myelopoiesis.

Four days after exposure, inflammation in the nasal cavity, lungs, lymph nodes, liver, and spleen continued to increase in severity and became more necrotizing. In the spleen, necrosis and/or depletion of white pulp lymphocytes was also identified. In the bone marrow, foci of histiocytic to fibrinous/necrotizing inflammation were identified in addition to the increased myeloid cells that were observed three days after exposure. Foci of necrotic cell debris were also identified within the epithelium and lamina propria of the jejunum. The gastrointestinal lesions were considered to be because of swallowing of bacteria after mucociliary clearance from the respiratory tract after aerosol exposure.

By day 5 after exposure and later, the inflammation in the primary affected tissues was primarily fibrinous and necrotizing (Figure 6). Additional foci of histiocytic to fibrinous/ necrotizing inflammation were sporadically identified in the adrenal glands, kidneys, and heart of some rats, presumably because of development of a secondary bacteremia. Isolated foci of alveolar septal wall necrosis, which were not associated with adjacent intra-alveolar inflammatory infiltrates, were identified in the lungs of many rats. In some rats that were found dead or euthanized near death, areas of hepatocellular coagulation necrosis were identified that were often associated with fibrin microthrombi in the hepatic

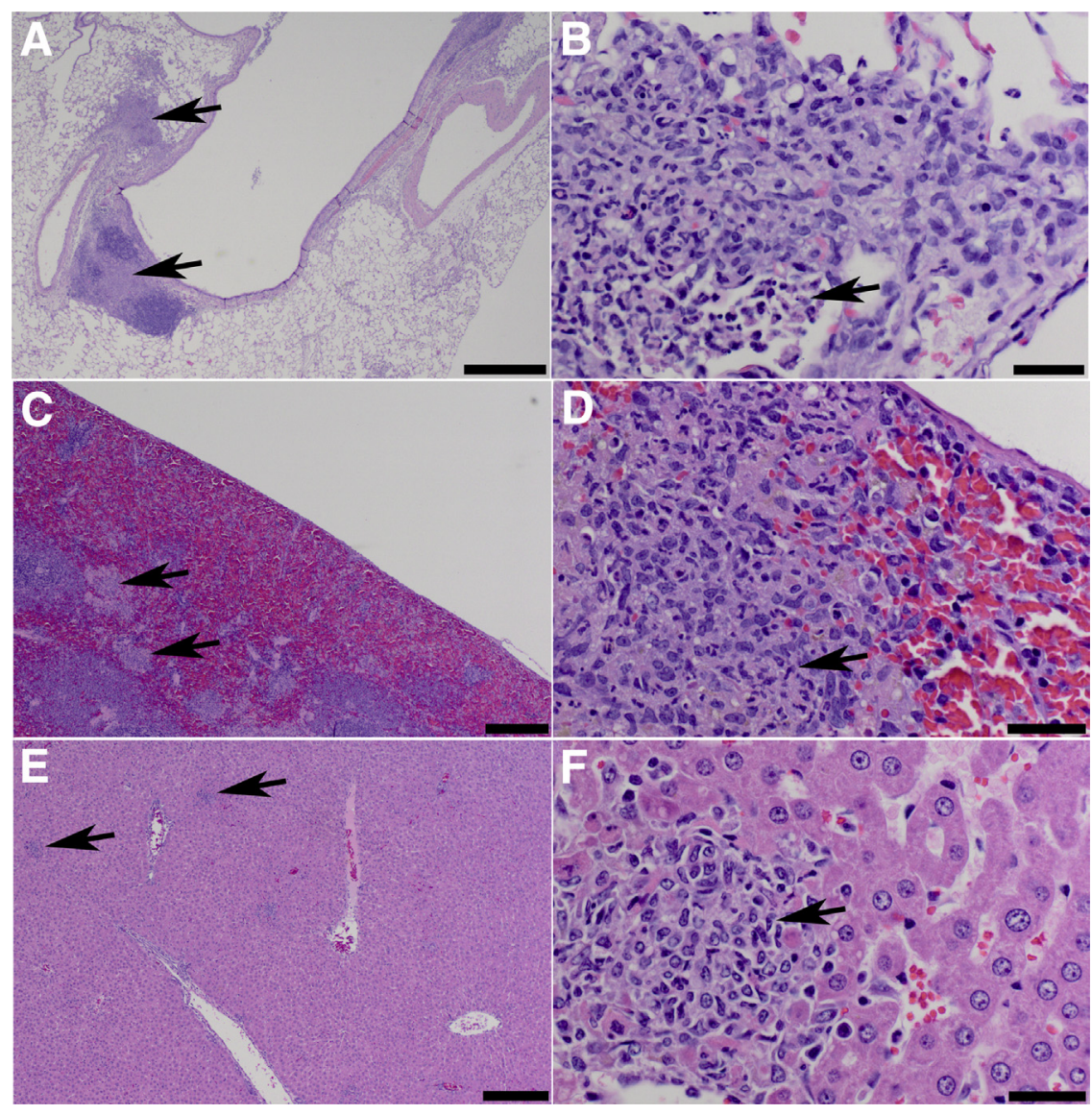

Figure 5 Neutrophilic and histiocytic infiltrates were identified in the lungs, spleen, and liver of F344 rats on day 3 after exposure to $10^{3}$ CFUs Francisella tularensis, strain SCHU S4. Inflammatory cell infiltrates (arrows) consisting of neutrophils and histiocytes were identified in the lungs ( $\mathbf{A}$ and $\mathbf{B}$ ), spleen ( $\mathbf{C}$ and $\mathbf{D})$, and liver ( $\mathbf{E}$ and $\mathbf{F}$ ) of F344 rats euthanized 3 days after challenge. In the lungs, inflammation was localized primarily to the conducting airways and centriacinar alveoli and, to a lesser extent, the vascular adventitia. In the spleen, the inflammation was randomly distributed as discreet foci in the red pulp. In the liver, the inflammation was randomly distributed as discreet foci within the hepatic parenchyma. Hematoxylin and eosin stain was used. Scale bars: $500 \mu \mathrm{m}(\mathbf{A})$; $20 \mu \mathrm{m}(\mathbf{B}, \mathbf{D}$, and $\mathbf{F}) ; 200 \mu \mathrm{m}$ (C and $\mathbf{E})$. 

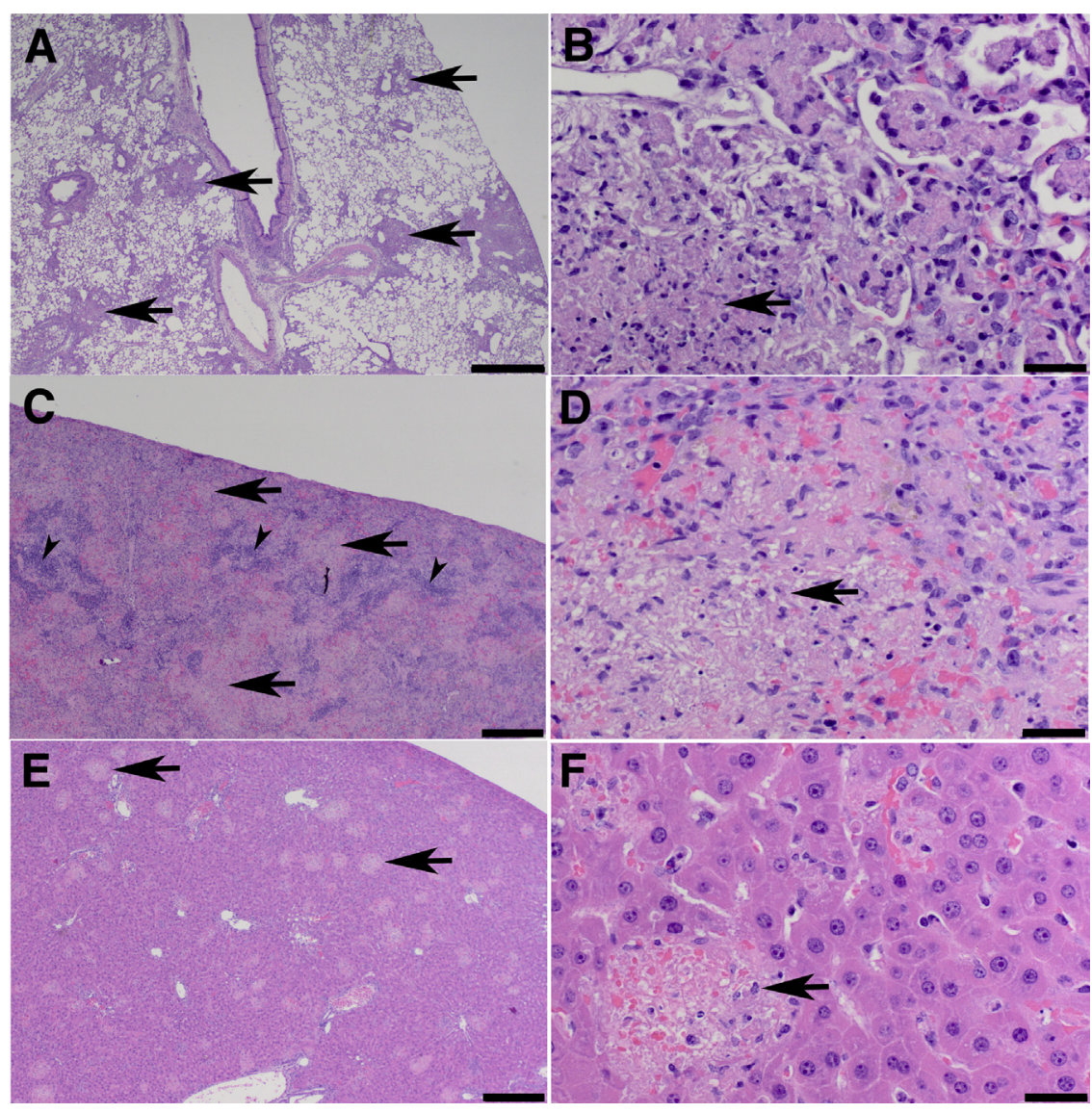

Figure 6 Inflammation in the lungs, spleen, and liver increased in severity and became more necrotizing over time after exposure to $10^{3}$ CFUs Francisella tularensis, strain SCHU S4. Inflammation (arrows) in the lungs ( $\mathbf{A}$ and $\mathbf{B}$ ), spleen (C and $\mathbf{D})$, and liver (E and $\mathbf{F}$ ) of a rat euthanized on day 7 after exposure involved more tissue than on day 3 and consisted primarily of histiocytes with fibrin and necrotic cell debris and fewer viable neutrophils. Inflammation in the splenic red pulp coalesced and effaced most of the red pulp and encroached on the white pulp, which was reduced in volume (arrowheads). Hematoxylin and eosin stain was used. Scale bars: $500 \mu \mathrm{m}$ (A); $20 \mu \mathrm{m}$ (B, D, and F); $200 \mu \mathrm{m}$ (C and $\mathbf{E})$.

sinusoids (Figure 7). Thrombi, with secondary mucosal edema, were also detected within the nasal mucosal vessels of several rats that were euthanized moribund between days 5 and 8 after exposure.

\section{Clinical Pathology}

Changes in select hematology parameters in the exposed rats are shown in Figure 8. Rats that were euthanized at scheduled time points on days 1 through 5 after exposure had progressively higher average neutrophil and monocyte counts than the naïve rats, consistent with the observation of inflammation in target tissues. In contrast, rats euthanized in a moribund condition between days 5 and 8 after exposure had average neutrophil and monocyte counts that had decreased to near the levels of the naïve rats. This may have been because of exhaustion of bone marrow progenitors or an imbalance between production and peripheral use of these cells as animals were near death. The average lymphocyte and platelet counts for the rats euthanized on day 1 after exposure were initially higher than for the naïve rats, but subsequently decreased progressively through scheduled euthanasia on day 5 and moribund euthanasia between days 5 and 8 . These decreases were considered to be a result of sepsis. Changes in other hematology parameters were unremarkable.

Changes in select clinical chemistry parameters in the exposed rats are shown in Figures 9 and 10. Increases in alanine and aspartate transaminase activities, alkaline phosphatase activity, and total bilirubin concentration were detected on day 3 after exposure and onwards and correlated with progressively increasing liver inflammation and necrosis. There were gradual and progressive decreases in albumin concentrations starting at day 1 after exposure, which were considered to be a result of decreased albumin synthesis as part of the negative acute phase response, possibly combined with albumin leakage into inflamed tissues. Calcium concentrations decreased in parallel with albumin concentrations. Some of the decrease in calcium concentrations was considered to be because of a decrease in the protein (albumin) bound fraction, and some was considered to be because of sepsis. ${ }^{27}$ Increases in $\alpha-2$ macroglobulin concentrations were first detected on day 3 after exposure, and these were markedly increased by day 5 after exposure. The increases in $\alpha$-2-macroglobulin were considered to be because of the acute phase response to F. tularensis.

Increases in blood urea nitrogen (BUN), creatinine, and phosphorus concentrations were noted starting on day 3 (BUN) and 4 (creatinine and phosphorus) after exposure and were interpreted to be secondary to dehydration and/or sepsis-induced alterations in renal perfusion. When rats were moribund, these increases were accompanied by elevations in chloride and potassium, which were considered to 

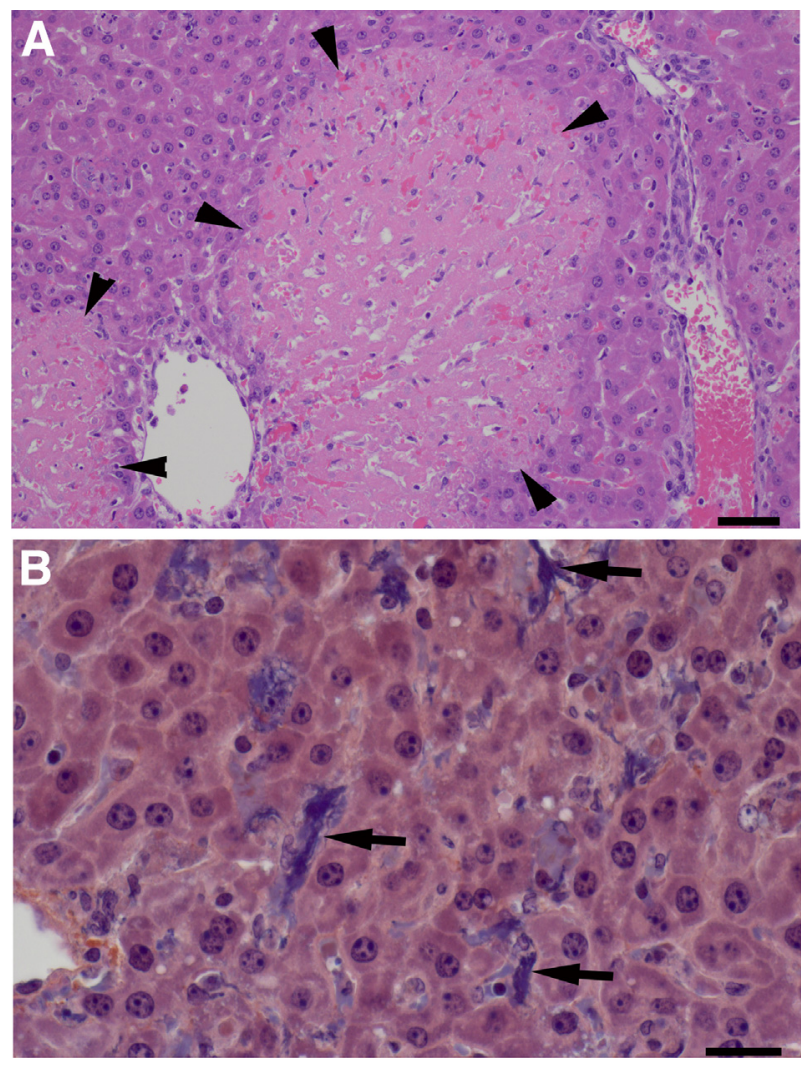

Figure 7 Hepatocellular coagulation necrosis and intrasinusoidal fibrin microthrombi were detected in the liver of some rats that were euthanized or found dead on or after day 5 after exposure to $10^{3}$ CFUs Francisella tularensis, strain SCHU S4. A: Hepatocellular coagulation necrosis (arrowheads) in a rat that was euthanized on day 7 because of progression of clinical disease. Hematoxylin and eosin stain was used. B: Fibrin microthrombi within hepatic sinusoids (arrows) of the liver of the rat in $\mathbf{A}$. Phosphotungstic acid hematoxylin stain was used. Scale bars: $50 \mu \mathrm{m}(\mathbf{A})$; $20 \mu \mathrm{m}(\mathbf{B})$.

be because of metabolic derangements and altered renal function due to sepsis. A gradual and progressive decrease in the average glucose concentration starting on day 2 after exposure was attributed to reduced food consumption and anorexia associated with infection combined with increased metabolic rate related to sepsis.

\section{Measurement of Fibrinogen and Clotting Times}

Hepatic microthrombi and hepatocellular coagulation necrosis, which were attributed to the development of disseminated intravascular coagulation, were identified in several rats that died or were euthanized after day 5 after exposure (Figure 7). To further characterize the natural history of inhalational tularemia in F344 rats and corroborate the development of disseminated intravascular coagulation as this disease progressed, an additional study was performed in which rats were exposed by inhalation to a target dose of $10^{3}$ CFUs (actual lung deposition, $330 \pm 54$ CFUs) of aerosolized SCHU S4. These rats were euthanized at predetermined time points to collect blood for measurement of fibrinogen and clotting times. Compared to naïve rats, fibrinogen was increased in two of four rats that were euthanized on day 3 after exposure (Table 2). Fibrinogen is a positive acute phase protein, so the initial increases in fibrinogen concentration were considered to be because of activation of the acute phase response in these rats. In the two rats with increased fibrinogen, prothrombin time and partial thromboplastin time were similar to the respective times observed for the naïve rats. In the other two of four rats euthanized on day 3 after exposure and in three of three and two of two rats euthanized on days 4 and 5 after exposure, respectively, fibrinogen was decreased compared to the naive control rats, whereas the prothrombin time and partial thromboplastin time for these rats were prolonged. The decrease in fibrinogen concentration and prolongation of clotting times were interpreted to be because of increased consumption of fibrinogen, and possible other clotting factors, associated with intravascular coagulation.

\section{Measurement of Lactate Levels}

Lactate is a marker of anaerobic metabolism, and in critically ill human patients, lactate levels $>4 \mathrm{mmol} / \mathrm{L}$ are considered to be an indicator of severe sepsis with organ hypoperfusion. To characterize the development and progression of sepsis in F344 rats as the disease progressed, jugular vein catheters were implanted in four rats, which were then exposed by inhalation to a target dose of $10^{3}$ CFUs (actual lung deposition, $3619 \pm 711$ CFUs) of aerosolized SCHU S4. Blood was collected through the catheters immediately before SCHU S4 exposure and then daily through day 5 after exposure for lactate assays. Serum from these rats was used to measure lactate levels over time, and the results are shown in Figure 11. Lactate levels increased progressively over the baseline levels from day 2 through day 4 after exposure, suggesting the development of severe sepsis during this time frame.

\section{Discussion}

Pneumonic tularemia is the most serious form of tularemia, and it is usually a result of inhalation of contaminated air. This can occur during natural exposure or intentional release during an act of war or terrorism. In preparation for these potential events, an enormous amount of resources have been allocated to the development of novel vaccines and therapeutics, all of which will need to be evaluated using well-characterized animal models because the natural occurrence of inhalational tularemia in humans is rare. Therefore, a useful small animal model for the development of therapeutics and vaccines should reproduce as many features of human disease pathogenesis and clinical presentation as possible. The F344 rat aerosol infection model was first developed by Dr. Joseph Jemski, who used it to evaluate the effect of vaccination route on the efficacy of 


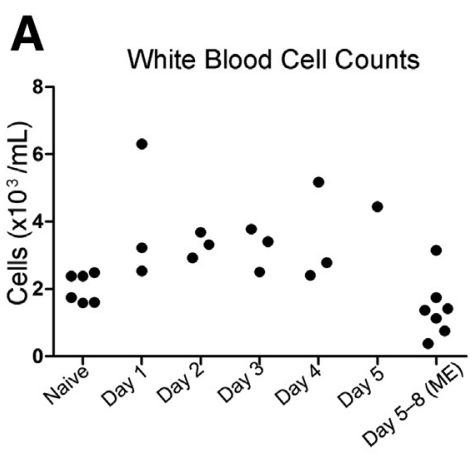

D

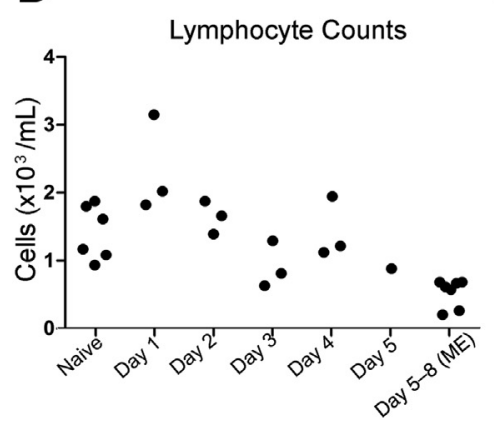

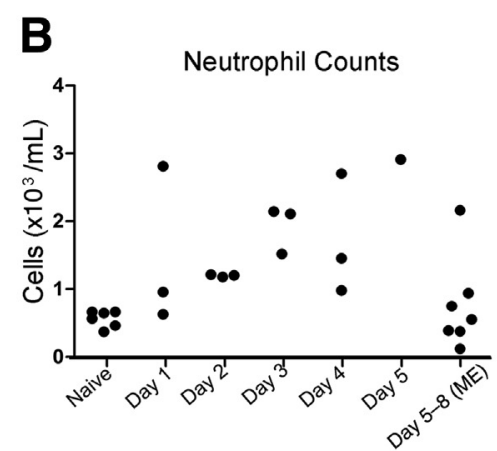

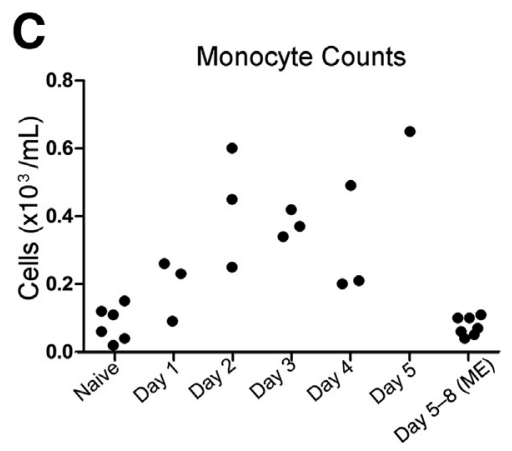

E

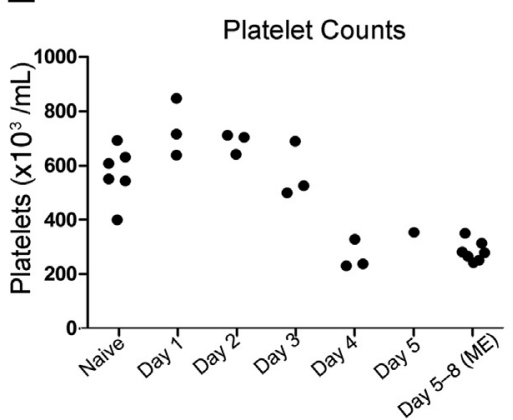

Figure 8 F344 rats exposed to $10^{3}$ CFUs SCHU S4 exhibited alterations in hematology parameters after exposure. Total white blood cells (A), neutrophils (B), monocytes (C), lymphocytes (D), and platelets (E). Each symbol represents the value from an individual rat on the designated day of euthanasia $(x$ axis) after exposure. The values for unexposed control (naïve) rats are also shown for comparison. ME, moribund euthanasia.

LVS in F344 rats. ${ }^{25}$ We have now extended his early work by showing that the natural history of pneumonic tularemia in female F344 rats bears close resemblance to what has been reported for the human disease. ${ }^{19,28-31}$

The initial sites of infection after aerosol exposure were the nasal cavity, lungs, and possibly the gastrointestinal tract. Gastrointestinal deposition is a common feature of inhalation exposures in multiple species, including humans, because of bacteria deposited in the pharynx being swallowed during the exposure or after mucociliary clearance of bacteria deposited in the respiratory tract. A previous single photon emission-computed tomographic/computed tomographic imaging study in F344 rats showed that up to $19 \%$ of radiolabeled polydisperse aerosols between 0.5 and $5 \mu \mathrm{m}$ in size were found in the stomach. ${ }^{32}$ It is unlikely that gastrointestinal tract infection contributed significantly to the pathologies we observed because SCHU S4 is much less infectious when it is ingested rather than inhaled $^{33}$; the pulmonary involvement occurred earlier and was more prominent than the gastrointestinal involvement; and diarrhea and gastrointestinal lesions were observed only when the animals became moribund.

The systemic dissemination of SCHU S4 from the lungs of rats that were exposed by aerosol inhalation to $10^{3}$ CFUs of $F$. tularensis was accompanied by increased neutrophil and monocyte counts, which were soon followed by development of a continuous fever and clinical signs of illness, with death occurring between days 5 and 8 after exposure. The time to fever onset was inversely correlated with the actual dose of SCHU S4 deposited in the lungs. The continuous fever pattern in the infected rats differed from the intermittent pattern that has been reported in human tularemia patients. The intermittent fever pattern may reflect control of systemic bacterial growth and release of bacterial products at various times in the disease process. ${ }^{34}$ Because most reported cases of tularemia in humans are caused by environmental exposure to presumably low doses of the bacteria, it is possible that a higher exposure dose or exposure to a more virulent strain of $F$. tularensis may overwhelm existing control mechanisms and cause humans to develop the same continuous fever pattern observed in the rats. Although dose-titration studies have been performed in humans, they were not designed to address this question because the patients were treated as soon as they became febrile.

Development of fever in F344 rats was accompanied by increases in mean arterial blood pressure and respiration rate. In all but 1 of the 20 infected rats monitored by telemetry, the onset of fever was not associated with a corresponding increase in heart rate. Such temperature-pulse dissociation, termed relative bradycardia in humans, has been reported in $42 \%$ of human patients with ulceroglandular tularemia or typhoidal tularemia. ${ }^{35}$ Relative bradycardia is not a defining feature of tularemia in people, but may be useful in differentiating tularemia from other infectious diseases that are not associated with relative bradycardia.

Activation of the acute phase response and increases in bilirubin and liver enzymes were first identified on day 3 

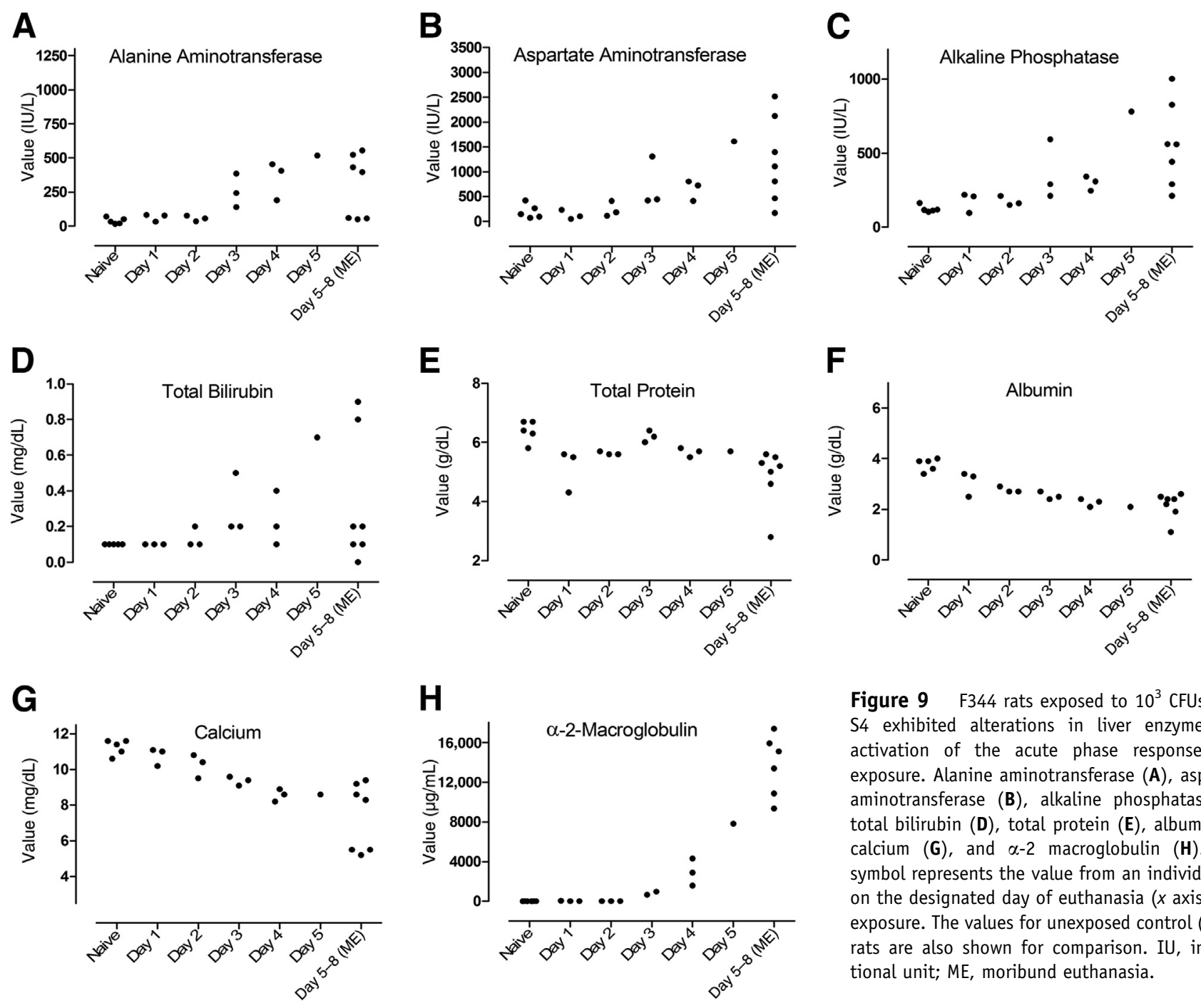

Figure 9 F344 rats exposed to $10^{3}$ CFUs SCHU S4 exhibited alterations in liver enzymes and activation of the acute phase response after exposure. Alanine aminotransferase $(\mathbf{A})$, aspartate aminotransferase (B), alkaline phosphatase (C), total bilirubin (D), total protein $(\mathbf{E})$, albumin $(\mathbf{F})$, calcium $(\mathbf{G})$, and $\alpha-2$ macroglobulin $(\mathbf{H})$. Each symbol represents the value from an individual rat on the designated day of euthanasia ( $x$ axis) after exposure. The values for unexposed control (naïve) rats are also shown for comparison. IU, international unit; $M E$, moribund euthanasia.

after exposure, similar to what has previously been reported in humans with tularemia. ${ }^{31,35-37}$ The progressive decreases in lymphocyte and platelet counts that were observed in this study, starting around day 3 after exposure, were considered to be because of sepsis. Decreases in lymphocyte and platelet counts have previously been reported in septic rats after cecal ligation and puncture and in humans from a variety of causes of sepsis. ${ }^{38-45}$ Changes in sodium, potassium, chloride, and bicarbonate were all unremarkable until the rats became moribund, at which point the chloride concentration was uniformly high, possibly because of sepsis-induced metabolic derangements and/or alterations in renal perfusion. BUN, creatinine, and phosphorus concentrations increased progressively over time in the rats in this study, which is consistent with the development of dehydration and/or alterations in renal perfusion because of sepsis. In human volunteers exposed to $F$. tularensis by the aerosol route, slight decreases in sodium and chloride and a slight increase in BUN were noted during the first few days after infection, but therapy was initiated when the first symptoms of illness appeared, so it is not known if these metabolic changes worsened over time. ${ }^{37}$ However, acute renal insufficiency with oliguria and azotemia has been reported as a terminal complication in 6 of 11 fatal cases of naturally occurring tularemia in humans. ${ }^{46}$

The results of the histopathology evaluation of tissues collected at predetermined time points after exposure and at moribund euthanasia or death showed that the affected organs and microscopic lesions in F344 rats were similar to those observed in humans with tularemia. ${ }^{1,47,48}$ Microscopic lesions in humans generally consist of neutrophilic and histiocytic infiltrates, which become progressively more fibrinous and necrotizing over time. After inhalation, primary lesions are described in the lungs and draining lymph nodes, with lymphohematogenous spread to organs that are rich in reticuloendothelial cells, most notably the liver, spleen, bone marrow, and lymph nodes. In the F344 rats exposed by inhalation to $10^{3}$ CFUs of aerosolized $F$. tularensis, the increasingly fibrinous and necrotizing 

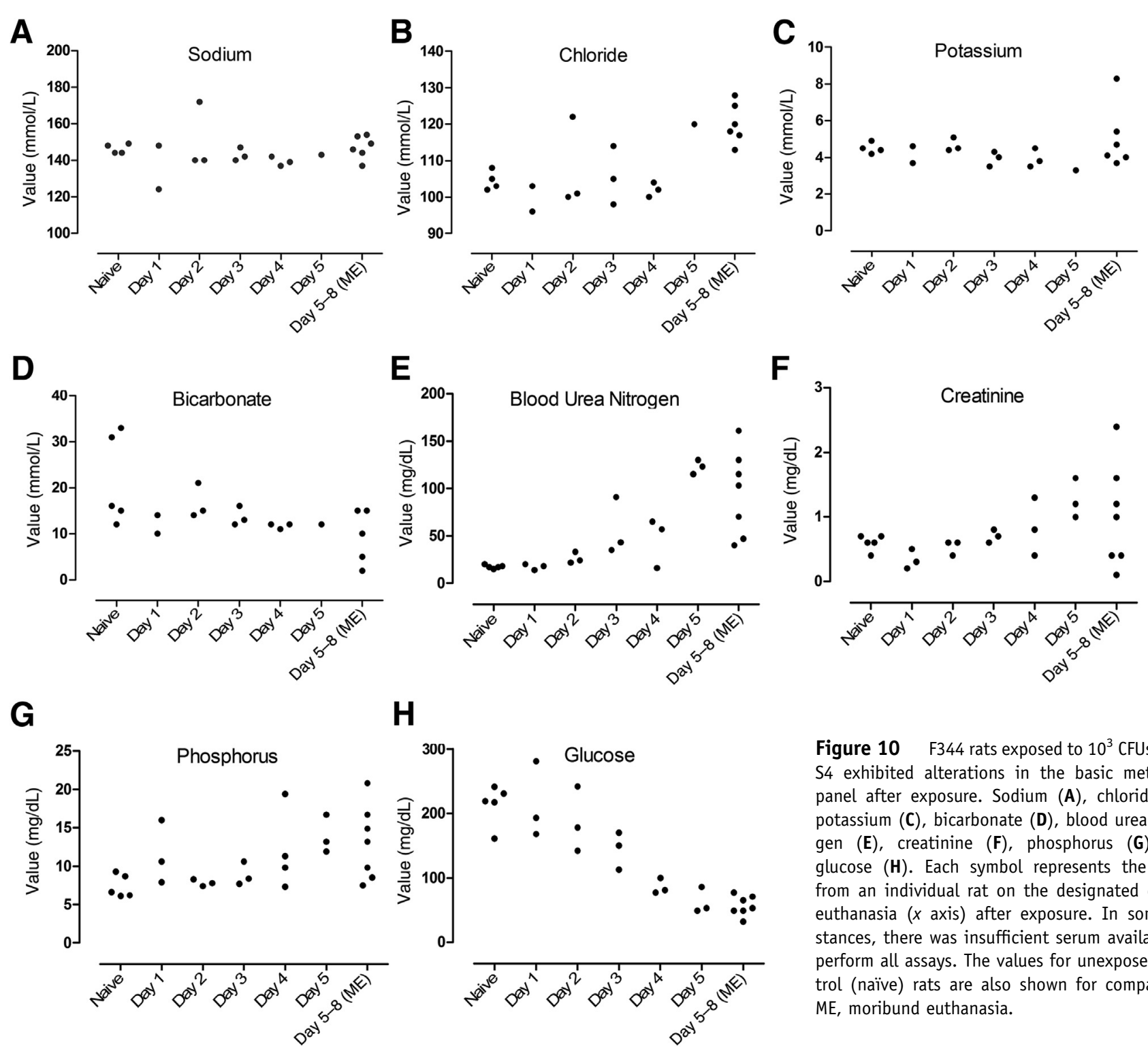

\begin{abstract}
Figure $10 \quad \mathrm{~F} 344$ rats exposed to $10^{3} \mathrm{CFUs}$ SCHU S4 exhibited alterations in the basic metabolic panel after exposure. Sodium (A), chloride (B), potassium (C), bicarbonate (D), blood urea nitrogen $(\mathbf{E})$, creatinine $(\mathbf{F})$, phosphorus $(\mathbf{G})$, and glucose $(\mathbf{H})$. Each symbol represents the value from an individual rat on the designated day of euthanasia ( $x$ axis) after exposure. In some instances, there was insufficient serum available to perform all assays. The values for unexposed control (naïve) rats are also shown for comparison. $M E$, moribund euthanasia.
\end{abstract}

inflammation in affected organs correlated with increasing organ bacterial burdens as the disease progressed.

In contrast to humans, none of the rats in this study exhibited pleuritis. The lack of pleuritis in rats was attributed to the anatomical differences in the pleural thickness between rats and humans. Rats have relatively thin pleura that is lacking in lymphatic vessels, whereas humans have thicker pleura with abundant lymphatic vessels. Lymphangitis, a common feature of $F$. tularensis during lymphatic transport of bacteria to draining lymph nodes, is presumably the source of pleural inflammation. The lack of lymphatics in the rat pleura likely was responsible for the absence of pleural inflammation.

When rats were in the late stages of the disease, sporadic inflammation was also detected in the adrenal glands, kidneys, and heart, presumably as a result of secondary bacteremia. Thrombosis, with coagulation necrosis in the liver and edema in the nasal cavity, was also identified in many rats that were near death. The development of thrombi in these organs was considered to be because of disseminated intravascular coagulation associated with severe sepsis.

The pathogenesis of pulmonary tularemia in the female F344 rat follows the continuum of clinical presentation and laboratory findings that characterize sepsis, severe sepsis, and septic shock in human patients. ${ }^{49}$ Sepsis in the infected rats was defined by fever, tachypnea, and leukocytosis observed 2 days after exposure. Severe sepsis was characterized by signs of hypoperfusion or end organ damage, including hypotension, elevated lactate, BUN, creatinine, liver enzymes, and bilirubin levels, thrombocytopenia, and coagulopathy. Although infected rats did not meet the strict definition of having septic shock because no attempt was made to resuscitate with fluids, septic shock and disseminated intravascular coagulation were considered to be the 
Table 2 PT, PTT, and Fibrinogen Concentration in Rats Exposed to Aerosolized Francisella tularensis, Strain SCHU S4

\begin{tabular}{lllll}
\hline \multirow{2}{*}{ Group } & Rat ID & $\begin{array}{l}\text { PT, } \\
\text { seconds }\end{array}$ & $\begin{array}{l}\text { PTT, } \\
\text { seconds }\end{array}$ & $\begin{array}{l}\text { Fibrinogen, } \\
\mathrm{mg} / \mathrm{dL}\end{array}$ \\
\hline Nä̈ve control & 1 & 15.1 & 30.9 & 244 \\
& 2 & 15.1 & 18.8 & 232 \\
& 3 & 15.1 & 45.2 & 262 \\
Day 3 after exposure & 4 & 14.7 & 34.1 & 230 \\
& 5 & 14.2 & 36.8 & 463 \\
& 6 & $>70$ & $>120$ & $<35.5$ \\
Day 4 after exposure & 9 & 45.9 & $>120$ & 114 \\
& 8 & 15.7 & 44.7 & 512 \\
& 10 & 31.8 & 47.9 & QNS \\
Day 5 after exposure & 11 & $>70$ & $>120$ & $<35.5$ \\
& 12 & $>70$ & $>120$ & $<35.5$ \\
& 14 & $>70$ & $>120$ & $<35.5$ \\
& 15 & $>70$ & $>120$ & QNS \\
\hline
\end{tabular}

ID, identification; PT, prothrombin time; PTT, partial thromboplastin time; QNS, quantity of serum not sufficient for analysis.

primary cause of death in these rats as in humans. Studies are underway to characterize the natural history of disease in rats challenged with a minimal lethal dose.

In addition to characterizing the pathogenesis of pneumonic tularemia in Fischer 344 rats, the development of animal models for testing the efficacy of therapeutics also requires the identification of a reliable marker of infection that can be used as a trigger to initiate treatment. Analysis of the data from these studies showed that the onset of fever in the rat model was the earliest, consistent, and readily detectable clinical indicator of bacteremia that would best mimic the stage of disease when patients are likely to present clinically, and thus would provide a reasonable trigger point for therapeutic intervention. The consistency of the time to fever onset across studies in which deposited dose ranged from 853 to 3153 CFUs is also beneficial in that the actual lung deposition can vary from target lung deposition by threefold when using aerosol delivery because of slight

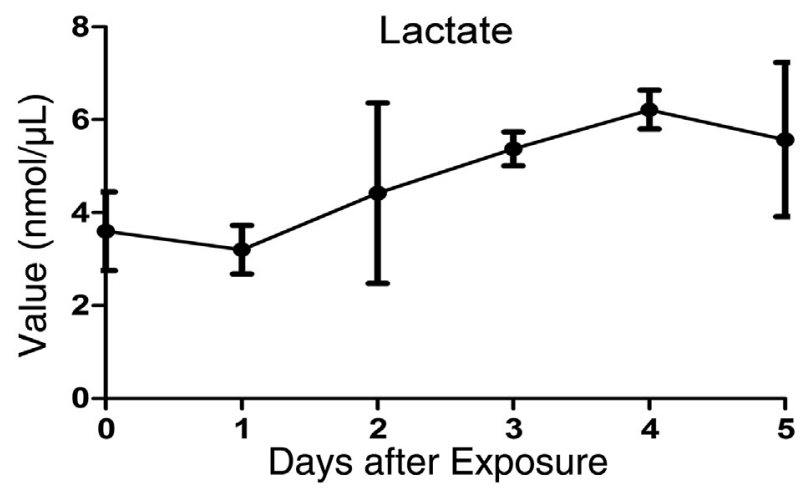

Figure 11 Lactate concentration increased over time in F344 rats challenged with $10^{3}$ CFUs SCHU S4. Data are presented as the means \pm SD from samples collected from the same four rats on different days. variations in generator concentrations and animal characteristics, such as breathing rate and pattern during exposure. Thus, the onset of fever will be a useful marker of infection in future studies testing therapeutics for efficacy against inhalational tularemia.

Although aerosol exposure recapitulates a natural infection route in humans, the equipment and the regulatory compliance required to safely perform this type of study make it inaccessible to many investigators. The alternative is to deliver a liquid bolus by intracheal or intranasal instillation. A direct comparison of aerosol, intratracheal, and intranasal delivery of $F$. tularensis in F344 rats has not been done in the same study. However, on the basis of previous studies in rats, the clinical progression, $\mathrm{LD}_{50}$, and terminal histopathology and bacterial burdens in the lungs, liver, and spleen appear to be similar whether $F$. tularensis is delivered via the intratracheal route or via small particle aerosol. ${ }^{23}$ However, one notable difference observed is that the aerosol inhalation method produces a more uniform distribution of lesions within the deep lung than intratracheal instillation. In addition, deposition of $F$. tularensis after aerosol exposoure was not only in the lungs but also in the nasopharyngeal region and the gastrointestinal tract, similar to what would be expected after its use as a bioweapon. There may be additional differences between aerosol and intratracheal exposure that will require more indepth characterization of the two models to discover. These distinctions are important considerations when the animal model will be used for pivotal studies to satisfy the Food and Drug Administration's Animal Efficacy Rule (21 CFR 314.610 and 21 CFR 601.91), which requires the animal model to reproduce the human disease as closely as possible. However, on the basis of our current understanding, there may be sufficient similarities between the two models that they can be used interchangeably for proof-ofconcept studies.

There is an extensive body of literature on murine models of pneumonic tularemia. Mice exposed to low doses $(<25$ CFUs) of $F$. tularensis subspecies tularensis by the intranasal and aerosol routes demonstrated similar clinical progression, terminal histopathology, and terminal bacterial burdens in the liver and spleen, but much less lung inflammation, than the F344 rats on this study. ${ }^{18,50,51}$ The low level of lung inflammation observed in these mouse studies may have been a result of the low exposure doses used. A more recent study using a higher aerosol exposure dose demonstrated many similarities in the natural history of pneumonic tularemia in mice and F344 rats infected with aerosolized $F$. tularensis subspecies tularensis, including the development of inflammation in the lungs, liver, and spleen with severe sepsis and coagulopathy, and high terminal bacterial burdens in these organs and blood at the time of death, 4 to 5 days after infection. ${ }^{52}$ The most prominent difference between F344 rats and mice is the susceptibility of the latter to all $F$. tularensis subspecies, including subspecies novicida and LVS. ${ }^{24}$ In this regard, F344 rats more 
closely resemble humans than do mice. This limitation of the murine model may cause many subunit vaccines that are less effective than LVS to be prematurely eliminated from further development. ${ }^{18}$

Overall, the female F344 rat model of inhalational tularemia closely resembles the human disease, and this should serve as a reliable, inexpensive animal model for studying pathogenic mechanisms and testing the efficacy of vaccines and therapeutics.

\section{Acknowledgments}

We thank Drs. Patrick Sanz, Tina Guina, Lynda Lanning, Lawrence Wolfraim, Mark Williams, and Rick Lyons for guidance on study design and critical review of the manuscript.

\section{Supplemental Data}

Supplemental material for this article can be found at http://dx.doi.org/10.1016/j.ajpath.2016.09.021.

\section{References}

1. Francis E, Callender GR: Tularemia: the microscopic changes of the lesions in man. Arch Pathol Lab Med 1927, 3:577-607

2. Saslaw S, Eigelsbach HT, Prior JA, Wilson HE, Carhart S: Tularemia vaccine study, II: respiratory challenge. Arch Intern Med 1961, 107: 702-714

3. McCrumb FR: Aerosol infection of man with Pasteurella tularensis. Bacteriol Rev 1961, 25:262-267

4. Hornick RB, Eigelsbach HT: Aerogenic immunization of man with live tularemia vaccine. Bacteriol Rev 1966, 30:532-538

5. Stuart BM, Pullen RL: Tularemic pneumonia: review of American literature and report of 15 additional cases. Am J Med Sci 1945, 210: $223-236$

6. Harris S: Japanese biological warfare research on humans: a case study of microbiology and ethics. Ann N Y Acad Sci 1992, 666: $21-52$

7. Christopher GW, Cieslak TJ, Pavlin JA, Eitzen EM Jr: Biological warfare: a historical perspective. JAMA 1997, 278:412-417

8. Alibek K: Biohazard. New York, Random House, 1999

9. Franz DR, Jahrling PB, Friedlander AM, McClain DJ, Hoover DL, Bryne WR, Pavlin JA, Christopher GW, Eitzen EM Jr: Clinical recognition and management of patients exposed to biological warfare agents. JAMA 1997, 278:399-411

10. Glynn AR, Alves DA, Frick O, Erwin-Cohen R, Porter A, Norris S, Waag D, Nalca A: Comparison of experimental respiratory tularemia in three nonhuman primate species. Comp Immunol Microbiol Infect Dis 2015, 39:13-24

11. Day WC, Berendt RF: Experimental tularemia in Macaca mulatta: relationship of aerosol particle size to the infectivity of airborne Pasteurella tularensis. Infect Immun 1972, 5:77-82

12. Schricker RL, Eigelsbach HT, Mitten JQ, Hall WC: Pathogenesis of tularemia in monkeys aerogenically exposed to Francisella tularensis 425. Infect Immun 1972, 5:734-744

13. Baskerville A, Hambleton P, Dowsett AB: The pathology of untreated and antibiotic-treated experimental tularaemia in monkeys. $\mathrm{Br}$ J Exp Pathol 1978, 59:615-623

14. Nelson M, Lever MS, Dean RE, Savage VL, Salguero FJ, Pearce PC, Stevens DJ, Simpson AJ: Characterization of lethal inhalational infection with Francisella tularensis in the common marmoset (Callithrix jacchus). J Med Microbiol 2010, 59:1107-1113

15. Nelson M, Lever MS, Savage VL, Salguero FJ, Pearce PC, Stevens DJ, Simpson AJ: Establishment of lethal inhalational infection with Francisella tularensis (tularaemia) in the common marmoset (Callithrix jacchus). Int J Exp Pathol 2009, 90:109-118

16. Twenhafel NA, Alves DA, Purcell BK: Pathology of inhalational Francisella tularensis spp. tularensis SCHU S4 infection in African green monkeys (Chlorocebus aethiops). Vet Pathol 2009, 46: 698-706

17. Lyons CR, Wu TH: Animal models of Francisella tularensis infection. Ann N Y Acad Sci 2007, 1105:238-265

18. Shen H, Chen W, Conlan JW: Susceptibility of various mouse strains to systemically- or aerosol-initiated tularemia by virulent type A Francisella tularensis before and after immunization with the attenuated live vaccine strain of the pathogen. Vaccine 2004, 22: 2116-2121

19. Saslaw S, Carhart S: Studies with tularemia vaccines in volunteers, III: serologic aspects following intracutaneous or respiratory challenge in both vaccinated and nonvaccinated volunteers. Am J Med Sci 1961, 241:689-699

20. Reed DS, Smith LP, Cole KS, Santiago AE, Mann BJ, Barry EM: Live attenuated mutants of Francisella tularensis protect rabbits against aerosol challenge with a virulent type A strain. Infect Immun 2014, 82:2098-2105

21. Baskerville A, Hambleton P: Pathogenesis and pathology of respiratory tularaemia in the rabbit. Br J Exp Pathol 1976, 57: 339-347

22. Reed DS, Smith L, Dunsmore T, Trichel A, Ortiz LA, Cole KS, Barry E: Pneumonic tularemia in rabbits resembles the human disease as illustrated by radiographic and hematological changes after infection. PLoS One 2011, 6:e24654

23. Wu TH, Zsemlye JL, Statom GL, Hutt JA, Schrader RM, Scrymgeour AA, Lyons CR: Vaccination of Fischer 344 rats against pulmonary infections by Francisella tularensis type A strains. Vaccine 2009, 27:4684-4693

24. Ray HJ, Chu P, Wu TH, Lyons CR, Murthy AK, Guentzel MN, Klose KE, Arulanandam BP: The Fischer 344 rat reflects human susceptibility to francisella pulmonary challenge and provides a new platform for virulence and protection studies. PLoS One 2010, 5: e9952

25. Jemski JV: Respiratory tularemia: comparison of selected routes of vaccination in Fischer 344 rats. Infect Immun 1981, 34:766-772

26. Pearse G: Histopathology of the thymus. Toxicol Pathol 2006, 34: 515-547

27. Muller B, Becker KL, Kranzlin M, Schachinger H, Huber PR, Nylen ES, Snider RH, White JC, Schmidt-Gayk H, Zimmerli W, Ritz R: Disordered calcium homeostasis of sepsis: association with calcitonin precursors. Eur J Clin Invest 2000, 30:823-831

28. James J, Kaul DR, Goldberger ZD, Saint S, Skerrett SJ: Clinical problem-solving: back to nature. N Engl J Med 2015, 373: $2271-2276$

29. Saslaw S, Carlisle HN: Studies with tularemia vaccines in volunteers, IV: Brucella aggiutinins in vaccinated and nonvaccinated volunteers challenged with Pasteurella tularensis. Am J Med Sci 1961, 242: 166-172

30. Dennis DT, Inglesby TV, Henderson DA, Bartlett JG, Ascher MS, Eitzen E, Fine AD, Friedlander AM, Hauer J, Layton M, Lillibridge SR, McDade JE, Osterholm MT, O'Toole T, Parker G, Perl TM, Russell PK, Tonat K: Tularemia as a biological weapon: medical and public health management. JAMA 2001, 285: $2763-2773$

31. Foshay L: Tularemia: a summary of certain aspects of the disease including methods for early diagnosis and the results of serum treatment in 600 patients. Medicine 1940, 19:1-84

32. Kuehl PJ, Anderson TL, Candelaria G, Gershman B, Harlin K, Hesterman JY, Holmes T, Hoppin J, Lackas C, Norenberg JP, Yu H, 
McDonald JD: Regional particle size dependent deposition of inhaled aerosols in rats and mice. Inhal Toxicol 2012, 24:27-35

33. KuoLee R, Zhao X, Austin J, Harris G, Conlan JW, Chen W: Mouse model of oral infection with virulent type A Francisella tularensis. Infect Immun 2007, 75:1651-1660

34. Ogoina D: Fever, fever patterns and diseases called "fever": a review. J Infect Public Health 2011, 4:108-124

35. Evans ME, Gregory DW, Schaffner W, McGee ZA: Tularemia: a 30year experience with 88 cases. Medicine (Baltimore) 1985, 64: 251-269

36. Matyas BT, Nieder HS, Telford SR 3rd: Pneumonic tularemia on Martha's Vineyard: clinical, epidemiologic, and ecological characteristics. Ann N Y Acad Sci 2007, 1105:351-377

37. Beisel WR, Sawyer WD, Ryll ED, Crozier D: Metabolic effects of intracellular infections in man. Ann Intern Med 1967, 67:744-779

38. Weber IB, Turabelidze G, Patrick S, Griffith KS, Kugeler KJ, Mead PS: Clinical recognition and management of tularemia in Missouri: a retrospective records review of 121 cases. Clin Infect Dis 2012, 55:1283-1290

39. Brooks HF, Osabutey CK, Moss RF, Andrews PL, Davies DC: Caecal ligation and puncture in the rat mimics the pathophysiological changes in human sepsis and causes multi-organ dysfunction. Metab Brain Dis 2007, 22:353-373

40. Drewry AM, Fuller BM, Skrupky LP, Hotchkiss RS: The presence of hypothermia within 24 hours of sepsis diagnosis predicts persistent lymphopenia. Crit Care Med 2015, 43:1165-1169

41. Drewry AM, Samra N, Skrupky LP, Fuller BM, Compton SM, Hotchkiss RS: Persistent lymphopenia after diagnosis of sepsis predicts mortality. Shock 2014, 42:383-391

42. Hotchkiss RS, Monneret G, Payen D: Sepsis-induced immunosuppression: from cellular dysfunctions to immunotherapy. Nat Rev Immunol 2013, 13:862-874

43. Claushuis TA, van Vught LA, Scicluna BP, Wiewel MA, Klein Klouwenberg PM, Hoogendijk AJ, Ong DS, Cremer OL, Horn J,
Franitza M, Toliat MR, Nurnberg P, Zwinderman AH, Bonten MJ, Schultz MJ, van der Poll T: Thrombocytopenia is associated with a dysregulated host response in critically ill sepsis patients. Blood 2016, 127:3062-3072

44. Vincent JL, Yagushi A, Pradier O: Platelet function in sepsis. Crit Care Med 2002, 30:S313-S317

45. Mavrommatis AC, Theodoridis T, Orfanidou A, Roussos C, Christopoulou-Kokkinou V, Zakynthinos S: Coagulation system and platelets are fully activated in uncomplicated sepsis. Crit Care Med 2000, 28:451-457

46. Giddens WR, Wilson JW Jr, Dienst FT Jr, Hargrove MD: Tularemia: an analysis of one hundred forty-seven cases. J La State Med Soc 1957, 109:93-98

47. Lamps LW, Havens JM, Sjostedt A, Page DL, Scott MA: Histologic and molecular diagnosis of tularemia: a potential bioterrorism agent endemic to North America. Mod Pathol 2004, 17:489-495

48. Guarner J, Zaki SR: Histopathology and immunohistochemistry in the diagnosis of bioterrorism agents. J Histochem Cytochem 2006, 54: $3-11$

49. American College of Chest Physicians/Society of Critical Care Medicine Consensus Conference: definitions for sepsis and organ failure and guidelines for the use of innovative therapies in sepsis. Crit Care Med 1992, 20:864-874

50. Conlan JW, Chen W, Shen H, Webb A, KuoLee R: Experimental tularemia in mice challenged by aerosol or intradermally with virulent strains of Francisella tularensis: bacteriologic and histopathologic studies. Microb Pathog 2003, 34:239-248

51. Wickstrum JR, Bokhari SM, Fischer JL, Pinson DM, Yeh HW, Horvat RT, Parmely MJ: Francisella tularensis induces extensive caspase-3 activation and apoptotic cell death in the tissues of infected mice. Infect Immun 2009, 77:4827-4836

52. Sharma J, Mares CA, Li Q, Morris EG, Teale JM: Features of sepsis caused by pulmonary infection with Francisella tularensis type A strain. Microb Pathog 2011, 51:39-47 